\title{
Los antiagregantes plaquetarios son eficaces para prevenir eventos cardiovasculares y reducir la mortalidad en pacientes con claudicación intermitente \\ Antiplatelet agents are effective to prevent cardiovascular events and reduce mortality in patients with
}

intermittent claudication

\section{Objetivo}

Sintetizar la evidencia científica sobre la eficacia de los antiagregantes antiplaquetarios (AGP) en la reducción de eventos cardiovasculares (infarto y accidente cerebrovascular), mortalidad por todas las causas y cardiovascular en pacientes con enfermedad arterial periférica (EAP) sintomáticos por claudicación intermitente $(\mathrm{Cl})$ estable, que no requieren intervención. Evaluar que AGP es más efectivo.

\section{Fuentes de datos}

Cochrane Peripheral Vascular Diseases Group Specialised Register y Cochrane Central Register of Controlled Trials (hasta 2011).

\section{Selección de estudios}

Ensayos dínicoscontrolados aleatorizados (ECCA) doble ciego que hubierancomparado AGP orales versus placebo o versus otros AGP en pacientes con CI estable con un seguimiento mínimo de 24 semanas realizados entre 1982 y 2004.
Wong PF y col. Cochrane Database Syst Rev. 2011 Nov 9;(11):CD001272.

\section{Extracción de datos}

Los datos fueron extraídos por dos revisores independientes, que recopilaron datos sobre la calidad metodológica, los participantes, las intervenciones y las medidas de resultado: mortalidad por todas las causas, cardiovascular, eventos cardiovasculares y eventos adversos.

\section{Resultados Principales}

Se reportaron resultados de 12.168 adultos (76,2\% varones; edad promedio 63,4 [21 a 80]). En 4.507 pacientes se comparó algún AGP (indobufeno [2], picotamida [2], ticlopidina [5], triflusal [1]) con placebo; y en 7.761 algún AGP (clopidogrel [1] y picotamida [1]) versus aspirina. Ver resultados en Tabla 1. La mortalidad cardiovascular solo fue reportada como punto final en las comparaciones de ticlopidina y placebo. Solo dos ensayos de ticlopidina reportaron eventos con sangrado mayor.

Tabla 1: AGP contra placebo y AGP no aspirina contra aspirina

\begin{tabular}{|c|c|c|c|c|}
\hline \multirow[t]{2}{*}{ Resultados } & \multirow[t]{2}{*}{$\mathrm{N}^{\circ}$ estudios } & \multicolumn{2}{|c|}{$\mathrm{N}^{\circ}$ pacientes } & \multirow[t]{2}{*}{ Riesgo relativo (IC95\%) } \\
\hline & & Intervención & Control & \\
\hline \multicolumn{5}{|l|}{ AGP contra placebo } \\
\hline Mortalidad por todas las causas & 5 & 1.959 & 1.967 & $0,76(0,60$ a 0,98$)$ \\
\hline Mortalidad cardiovascular & 4 & 809 & 813 & $0,54(0,32$ a 0,93$)$ \\
\hline Eventos cardiovasculares & 5 & 1.959 & 1.967 & $0,80(0,63$ a 1,01$)$ \\
\hline Sangrado mayor & 2 & 422 & 416 & $1,73(0,51$ a 5,83$)$ \\
\hline Suspensión por event. adv. & 8 & 2.184 & 2.204 & $2,05(1,53$ a 2,75$)$ \\
\hline Síntomas gastrointestinales & 9 & 1.899 & 1.919 & $2,11(1,23$ a 3,61$)$ \\
\hline \multicolumn{5}{|l|}{ AGP no aspirina contra aspirina } \\
\hline Mortalidad por todas las causas & 2 & 3.826 & 3.835 & $0,73(0,58$ a 0,93$)$ \\
\hline Mortalidad cardiovascular & 2 & 3.826 & 3.835 & $0,74(0,48$ a 1,15$)$ \\
\hline Eventos cardiovasculares & 2 & 3.826 & 3.835 & $0,81(0,67$ a 0,98$)$ \\
\hline Sangrado mayor & 1 & 603 & 606 & $0,14(0,02$ a 1,16$)$ \\
\hline Suspensión por event. adv. & 1 & 603 & 606 & $0,83(0,62$ a 1,11$)$ \\
\hline Síntomas gastrointestinales & 1 & 603 & 606 & $0,60(0,45$ a 0,79$)$ \\
\hline
\end{tabular}

Conclusiones

En pacientes con EAP con Cl estable la utilización de AGP reduce la mortalidad general y la cardiovascular en comparación con placebo. Al comparar aspinina con clopidogrel o picotamida, estos últimos demostraron mayor reducción de mortalidad por todas las causas y menor incidencia de eventos cardiovasculares. En los pacientes tratados contra placebo se observaron mayores síntomas gastrointestinales y otros EA que obligaron a intemumpir la medicación. No se puede sacar conclusiones del sangrado mayor por ser escaso el número de pacientes en el estudio reportado.

Conflicto de interés de los autores: No presentan.

\section{Comentario}

La Cl es la manifestación más frecuente de la enfermedad arterial periférica ( $40 \%$ de los pacientes), con una prevalencia de entre 5 hasta un $20 \%$ en mayores de 70 años. Además de que empeora la calidad de vida del paciente, es un factor de riesgo independiente para eventos cardiovasculares. El objetivo del tratamiento con AGP es reducir los eventos isquémicos y muerte de origen cardiovascular. Las guías de práctica clínica recomiendan su uso, si bien no hay un acuerdo en que AGP seleccionar ${ }^{1-4}$. Los resultados de esta revisión coinciden con el informe del Estudio Colaborativo con Antitrombóticos, que reveló una reducción de un $23 \%$ en la variable final combinada de muerte cardiovascular, infarto o accidente cerebrovascular en los pacientes con EAP que fueron tratados con AGP (fundamentalmente aspirina) en comparación con el placebo ${ }^{56}$. Debemos realizar algunas consideraciones relacionadas con esta revisión: los datos analizados corresponden a pacientes con $\mathrm{Cl}$ estable por lo que no pueden ser extrapolados a otros

Claudia Paviotti [ ACA Salud claupaviotti@gmail.com]

Paviotti C. Los antiagregantes plaquetarios son eficaces para prevenir eventos cardiovasculares y reducir la mortalidad en pacientes con claudicación intermitente. Evid Act Práct Ambul. 2014, 17(2). Abr-Jun. 53. Comentado de: Wong PF, Chong LY, DP. Antiplatelet agents for intermittent claudication. Cochrane Database Syst Rev. 2011 Nov 9;(11):CD001272. doi: 10.1002/14651858.CD001272.pub2. PMID: 22071801.

\section{Referencias}

1. Alonso-Coello P, y col. American College of Chest Physicians Antithrombotic therapy in peripheral artery disease. Chest 2012; 141(2)(suppl):e669S-e690S.

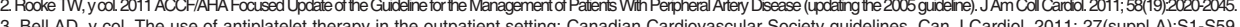

3. Bell AD, $y$ col. The use of antiplatelet therapy in the outpatient setting: Canadian Cardiovascular Society guidelines. Can J Cardiol. 2011; 27(suppl A):S1-S59.

5. Antithrombotic Trialists' Collaboration. Collaborative meta-analysis of randomised trials of antiplatelet therapy for prevention of death, myocardial infarction, and stroke in high risk patients. BMJ. 2002;324(7329):71-86. 6. Catalano M., y col. Prevention of serious vascular events by aspinin amongst patients with peripheral arterial disease: randomized, double-blind trial. J Intem Med. 261 2007:276-284. 